\title{
Soft X-ray Characterization Technique for Li Batteries under Operating Conditions
}

\author{
Cole F.Petersburg ${ }^{\mathrm{a}}$ Robert C.Daniel $^{\mathrm{a}}$, Cherno Jaye $^{\mathrm{b}}$, Daniel A.Fischer ${ }^{\mathrm{b}}$ Faisal M. Alamgir $^{\mathrm{a}}$ \\ ${ }^{\text {a }}$ School of Materials Science and Engineering, Georgia Institute of Technology, Atlanta, GA 30332,

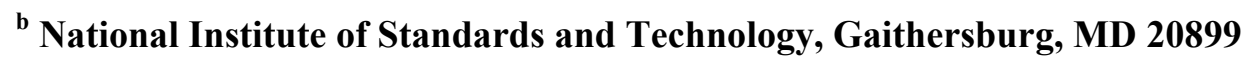

Synopsis A novel solid state lithium battery design enables surface- and bulk-sensitive soft X-ray absorption spectroscopy of battery materials under operating conditions.

Abstract $\mathrm{O}$ K edge and Co L edge near edge X-ray absorption fine structure (NEXAFS) was used to examine the cathode of an intact solid-state lithium ion battery. The novel technique allowed for the simultaneous acquisition of partial electron yield and fluorescence yield data during the first charge cycle of a $\mathrm{LiCoO}_{2}$-based battery below the intercalation voltage. The chemical environments of oxygen and cobalt at the surface are shown to differ chemically from those in the bulk. The present design enables a wide variety of in situ spectroscopies, microscopies, and scattering techniques.

\section{Keywords: NEXAFS, XANES, Li ion battery, in situ, $\mathrm{LiCoO}_{2}$}

\section{Introduction}

The excellent energy density (Tarascon et al. 2001) of rechargeable lithium batteries make them the preeminent energy storage technology for portable electronics and electric vehicles. Still, the safety and cycle life of intercalation cathodes are in doubt. Without direct measurements of the intercalation reaction as a function of cell potential over multiple cycles, selection of the best cathode material is guesswork. While it is known that a lithium ion leaving the cathode, upon charging, must be balanced by the loss of an electron, it is not known whether this electron comes from the transition metal ion alone or also from oxygen. Even for the dominant commercial cathode material, $\mathrm{LiCoO}_{2}$, it is not clear whether the electron is taken from cobalt to form a $\mathrm{Co}^{4+}$ ion or from oxygen to produce $\mathrm{O}^{-}$ions or oxygen gas (Dahn et al. 1994). Although $\mathrm{Co}^{4+}$ has been detected by EPR in coordination complexes (Koikawa et al. 1989), Co(IV) compounds are quite rare in nature and therefore may be highly reactive in a charged lithium battery. For mixed transition metal oxides, the picture is even more complex and interesting because multiple $3 \mathrm{~d}$ metals with multiple oxidation states are reacting simultaneously, and influencing each other (Deb et al. 2004). Any additional electron donation by oxygen ions would result in highly reactive species which may encourage the gradual combustion, or the violent explosion, of nonaqueous electrolytes. A method for detecting gas evolution and measuring the oxidation state of oxygen and the $3 \mathrm{~d}$ transition metals in situ is presented.

Battery researchers have long sought to characterize cathode materials with X-rays in situ rather than ex situ because the requisite disassembly and washing of a cathode may change the bulk or surface composition significantly and eliminate internal 
electric fields. Furthermore, only one sample need be prepared to characterize an intercalation cathode at multiple cell potentials which not only reduces the work required, but also eliminates variations due to sample preparation. Ex situ methods become cumbersome when investigating the effects of multiple cycles. Only in situ cells can be measured over multiple cycles, enabling life cycle studies of cathodes. Extensive in situ X-ray Absorption Near Edge Spectroscopy (XANES) work has successfully characterized these materials at the metal $\mathrm{K}$ edges using hard $\mathrm{X}$-ray energies above $4 \mathrm{keV}$ with the use of Kapton windows and standard nonaqueous electrolytes. With this method, the oxidation states of transition metal ions in intercalation cathodes have been characterized as a function of state-of-charge (Deb et al. 2004; Alamgir et al. 2005). In these studies, a shift in the XANES spectra to higher energies is attributed partly to an increase in the $1 \mathrm{~s}$ binding energy due to a loss of $3 \mathrm{~d}$ electrons which would otherwise shield the nucleus.

Unfortunately, the dipole selection rule allows only $1 \mathrm{~s} \rightarrow 4 \mathrm{p}$ transitions at the $\mathrm{K}$ edge. The occupancy, configurations and energies of the $3 \mathrm{~d}$ orbitals of the first row transition metals, which form bonds with $\mathrm{O}$ in most Li battery cathodes, are not measured. The L edge spectra, by contrast, arise due to $2 p \rightarrow 3 d$ transitions. The amplitudes of some near edge features are proportional to the number of empty $3 \mathrm{~d}$ states, while changes in the $2 \mathrm{p}$ binding energy still cause energy shifts in the spectra. In situ XANES (called NEXAFS, Near Edge X-ray Absorption Fine Structure in the soft-X-ray context) of transition metal L edges can provide more direct oxidation information than can $\mathrm{K}$ edge XANES. In addition, hard X-ray in situ studies are seldom surface sensitive; the present method can show surface-to-bulk differences at the metal $\mathrm{L}$ edges that could not be seen at the $\mathrm{K}$ edges.

Additionally, soft X-ray NEXAFS spectroscopy accesses the $1 \mathrm{~s} \rightarrow 2 \mathrm{p}$ transitions of lighter elements including C, O, S, and P. The loss of $\mathrm{O} 2 \mathrm{p}$ electrons on deintercalation has been speculated to contribute to the capacity of $\mathrm{LiCoO}_{2}$ (Montoro et al. 2000; Yoon et al. 2002; Alamgir et al. 2005). Theoretical calculations (Ceder et al. 1998) and hard X-ray EXAFS experiments (Alamgir et al. 2005) suggest that oxygen contributes some of the capacity of $\mathrm{LiCoO}_{2}$, possibly by forming $\mathrm{O}^{-}$ions during the deintercalation of lithium. Also, the irreversible formation of $\mathrm{Li}_{2} \mathrm{O}$ can be detected by its characteristic absorption spectrum. NEXAFS at the O K edge, therefore, provides a great deal of information on intercalation reactions. Many ex situ studies (Montoro et al. 2000; Uchimoto et al. 2001; Yoon et al. 2002), have shown $\mathrm{LiCoO}_{2}$ and other cathodes at multiple states of charge by chemically or electrochemically adjusting the lithium content. Changes in both the oxygen $\mathrm{K}$ edge and transition metal $\mathrm{L}$ edges have been detected as a function of lithium content, but without the electric fields of a battery. These studies have been unable to show oxygen's $\mathrm{K}$ edge spectrum in an assembled battery due to design difficulties. $\mathrm{X}$-rays below $1 \mathrm{keV}$ in energy have attenuation lengths of $\sim 500 \mathrm{~nm}$ in solid materials; such a thin X-ray window cannot contain the pressure of a liquid electrolyte. Instead, a vacuum-compatible cell is required.

Only an in situ soft X-ray electrochemical cell, lacking windows or liquids, allows for surface sensitive and bulk sensitive spectroscopy over a continuous range of cell voltages and at multiple cycles. Recent advances in X-ray focussing can even enable spectromicroscopy at solid electrochemical interfaces on the top surface and along the outer profile of the cell. The exposure of the cell to vacuum allows mass spectroscopic methods and gas-exposure experiments. For example, the effect of water vapor and oxygen on the electronic structure of the cathode and anode at various voltages can be studied in such a packaging-free cell by adjusting the partial pressures of these gases in the vacuum chamber. 
In the present study, surface-sensitive and bulk-sensitive NEXAFS measurements of the cathode of an intact, packaging-free solid state lithium battery are presented. Partial electron yield and fluorescence yield techniques reveal that the chemical environments of oxygen and of cobalt at the surface differ from those in the bulk in $\mathrm{LiCoO}_{2}$. The cell was successfully charged in a vacuum synchrotron endstation under high vacuum conditions with simultaneous collection of surface-sensitive and bulksensitive signals. This is the first-ever reported in situ soft X-ray absorption study of a working lithium battery.

\section{Experiment}

\subsection{Cell Design and Assembly}

The solid state lithium battery consisted of a three-layer powder compact, shown schematically in Figure 1b. The cathode layer consisted of a mixture of $14 \mathrm{wt} \% \mathrm{LiCoO}_{2}(7-10 \mu \mathrm{m}$, Sigma-Aldrich) $41 \mathrm{wt} \%$ solid electrolyte powder and $45 \mathrm{wt} \%$ acetylene black (Sigma-Aldrich) mixed in a SPEX 8000 planetary ball mill but without grinding media. The electrolyte layer consisted of partially amorphized lithium phosphorous sulfide glass of the formula $75 \mathrm{Li}_{2} \mathrm{~S} \cdot 25 \mathrm{P}_{2} \mathrm{~S}_{5}$. The electrolyte was mixed in an argonfilled glovebox, vacuum sealed into an agate vial equipped with Viton ${ }^{\mathrm{TM}}$ o-rings and three 12 mm agate balls, and amorphized in a SPEX 8000 mill for 20 hours. XRD showed the powder to contain a large amorphous fraction, indicating its lithium ion conductivity (Hayashi et al. 2001).

The cathode and electrolyte layers were pressed in a $13 \mathrm{~mm}$ die at $2050 \mathrm{~kg} / \mathrm{cm}^{2}$ in an argon-filled glovebox containing less than $1 \mathrm{ppm}$ of moisture. An $11 \mathrm{~mm}$ diameter piece of scraped lithium foil served as the anode. A pair of aluminum plates served as current collectors. An $8 \mathrm{~mm}$ countersunk hole in the cathode current collector allowed for a wide collection angle. A shallow counterbore held the pellet in position while nylon screws held the current collector plates together. Figure 1a shows the assembly without the screws. The assembly was inserted into the loadlock of the analysis chamber at the beamline via a nitrogenfilled glovebag. 


\subsection{Electrochemical Cycling}

The battery was transferred from the loadlock to the soft $\mathrm{x}$-ray analysis chamber and pumped down to $3 \times 10^{-8}$ torr. It was charged via a feedthrough by a Solartron 1287 potentiostat. The initial voltage of the as-prepared battery was $1.9 \mathrm{~V}$. It was later charged at 10 microamps to $2.5 \mathrm{~V}$ for $10.5 \mathrm{~h}$, followed by a second charging to $3.15 \mathrm{~V}$ at 25 microamps for $5 \mathrm{~h}$. At all times the battery remained below the onset voltage of approximately 3.9V. (Alamgir et al. 2005)

\subsection{X-ray Absorption Measurements}

NEXAFS spectra were taken at the NIST soft x-ray beamline U7A of the National Synchrotron Light Source at Brookhaven National Laboratory. The incident beam irradiated the sample at $38^{\circ}$ from the sample normal. Data were simultaneously collected in $0.2 \mathrm{eV}$ steps from a partial electron yield (PEY) detector, held $35^{\circ}$ below the sample normal, and from a fluorescence yield detector positioned perpendicular to the incident beam, or $52^{\circ}$ from the sample normal. The PEY detector was biased at $-250 \mathrm{~V}$ to reject low-energy electrons. The fluorescence yield (FY) data were filtered to include only O K edge transitions, or only the fluorescence from cobalt in Co L edge spectra. A clean, high-transmittance gold mesh was used to calibrate the oxygen spectra and to normalize spectral intensity while an FeNiCrCo alloy mesh was used to calibrate cobalt spectra.

\section{Results}

NEXAFS spectra at the O K edge in Figure 2a, in both PEY and FY modes, show a sharp resonance at $530 \mathrm{eV}$ and broad pair of resonances around $540 \mathrm{eV}$ with a shoulder feature at $\sim 535 \mathrm{eV}$. Relative to the PEY spectrum, the FY spectrum contains a more intense $530 \mathrm{eV}$ resonance and a stronger $540 \mathrm{eV}$ resonance. The shoulder resonance at $535 \mathrm{eV}$ is lower in energy in the bulk-sensitive FY spectrum than in the PEY spectrum.

Figure 3 shows the $\mathrm{O} \mathrm{K}$ edge at its initial voltage of $1.9 \mathrm{~V}$, and also while charging the cell past $2.3 \mathrm{~V}$ and $3.1 \mathrm{~V}$. A small feature at $533 \mathrm{eV}$ was seen when no current was flowing at cell potentials of $1.9 \mathrm{~V}$ and at $2.5 \mathrm{~V}$. While charging, the feature was temporarily reduced in size.

Co $\mathrm{L}$ edge spectra in Figure $2 \mathrm{~b}$ show more dramatic peak height differences between the surface-sensitive PEY data and the bulk-sensitive FY data. The peak area ratios $\left(\mathrm{L}_{3}\right.$ vs. $\left.\mathrm{L}_{2}\right)$ also differ; PEY scans show the 2:1 area ratio, which was expected based on spin considerations (Leapman et al. 1982), while FY scans indicate a 1.5:1 ratio. Additionally, the FY L peaks are broader than the PEY peaks.

\section{Discussion}

Soft X-ray NEXAFS indicates that no permanent chemical changes occur between $1.9 \mathrm{~V}$ and $3.15 \mathrm{~V}$. This is in accordance with cyclic voltammetry data (Alamgir et al. 2005) showing that intercalation begins at $3.9 \mathrm{~V}$ or higher in $\mathrm{LiCoO}_{2}$. The present design will allow for extensive in situ studies at higher voltages and over the course of many cycles. However, the present samples utilized an incompletely amorphized solid electrolyte, leading to a very high internal resistance in the cell. Together with an imperfect electrolyte-lithium-metal interface, the poorly-amorphized solid electrolyte limited the speed of operation of the battery. In the best case, the sulfide electrolyte conductivity has been reported to reach $2.010^{-4} \mathrm{~S} / \mathrm{cm}$ at $75 \% \mathrm{Li}_{2} \mathrm{~S} \cdot 25 \% \mathrm{P}_{2} \mathrm{~S}_{5}$. 
(Hayashi et al. 2001) Polyethylene oxide composites can reach $1.4 \times 10^{-4} \mathrm{~S} / \mathrm{cm}$ (Pitawala et al. 2008), but only with the addition of lithium salts, $15 \%$ ceramic filler as well as $50 \mathrm{wt} \%$ aprotic solvents, making them unsuitable for vacuum work.

The pre edge peak corresponds to molecular orbitals formed from O $2 \mathrm{p}$ and Co $3 \mathrm{~d}$ orbitals, while the $540 \mathrm{eV}$ peaks represent transitions from O 1s to molecular orbitals between O 2p and hybridized Co 4sp orbitals. (de Groot et al. 1989) The Co L edge is split into the $\mathrm{L}_{3}(780 \mathrm{eV})$ and $\mathrm{L}_{2}(\sim 795 \mathrm{eV})$ resonances, both of which correspond to transitions from Co $2 \mathrm{p}$ states to O $2 \mathrm{p}-\mathrm{Co} 3 \mathrm{~d}$ molecular orbitals.

Historically, soft X-ray studies have used ex situ samples and total electron yield detection (Montoro et al. 2000; Uchimoto et al. 2001; Yoon et al. 2002), in which the drain current resulting from photoemission is measured. This method suffers from background noise and a lack of data from the bulk. In partial electron yield detection, a bias grid reduces the background by rejecting low energy electrons from other elements in the sample. PEY and TEY are surface sensitive due to the low inelastic mean free path of electrons.

Oxygen K edge NEXAFS data show that, in the bulk, the O 2p-Co $3 \mathrm{~d}$ molecular orbitals are less filled than at the surface. In the bulk, this resonance is broader, indicating that a wider variety of energy levels is present. Separately, the O $2 p-C o ~ 4 s p$ molecular orbitals are more filled in the bulk than at the surface, leaving fewer unoccupied bound states for X-ray absorption. Because the cell was below the deintercalation onset potential, these differences are likely due to a difference in bond symmetry at the surface. However, transient effect was seen wherein a small resonance at $533 \mathrm{eV}$ was suppressed by the flow of current through the cell. This effect proves that the proportions of conductive additives and solid electrolyte in the composite cathode layer are at least sufficient for battery operation, if not more so. Also, such changes to the electronic structure of oxygen may tend to stabilize or destabilize oxygen ions in the lattice of $\mathrm{LiCoO}_{2}$, thereby affecting oxygen gas emissions.

Both FY and PEY O K edge spectra differ considerably from ex situ results in TEY mode in that the pre edge peak is less intense than the post edge peaks. While the spectra in Figure 2 resolve the peak at $540 \mathrm{eV}$, the feature at $536 \mathrm{eV}$ appears as only a thin shoulder on the peak. To date, only one bulk sensitive ex situ spectrum has shown the oxygen $\mathrm{K}$ edge in $\mathrm{Li}_{\mathrm{x}} \mathrm{CoO}{ }_{2}$ where $x$ was 0.35 (Yoon et al. 2004).

Yoon et al. (2004) found that in PEY spectra of $\mathrm{Li}_{0.4} \mathrm{Mn}_{2} \mathrm{O}_{4}$, the broad peaks near 540 eV were lower in intensity compared to the $530 \mathrm{eV}$ peak. Simultaneously collected FY spectra showed the $530 \mathrm{eV}$ peak to have a comparable intensity to the broad peaks near $540 \mathrm{eV}$; in this work, the same surface-to-bulk trend is seen in $\mathrm{LiCoO}_{2}$. Yoon et al. (2007) found the same trend of a reduced peak height in the bulk of $\mathrm{LiNi}_{0.8} \mathrm{Co}_{0.15} \mathrm{Al}_{0.05} \mathrm{O}_{2}$, yet bulk-sensitive data on $\mathrm{LiCoO}_{2}$ has been missing from the literature until now.

Cobalt L edge fluorescence yield data is vulnerable to self absorption whereby the photons emitted from the bulk are partly absorbed before they escape the sample. Because absorption is nonlinear, taller peaks such as the $\mathrm{L}_{3}$ tend to be attenuated more than would the $\mathrm{L}_{2}$. This may explain the discrepancy in peak area ratios as well as the lower peak intensities in fluorescence yield data. However, the broader FY peaks are indicative of a wider range of transition energies in the bulk compared to the surface. On the other hand, deviations in the expected 2:1 peak area ratios have been observed for several transition metals and their oxides. The ratio is influenced by the $3 \mathrm{~d}$ band occupancy, and is therefore sensitive to the chemical state of the transition metal ion. (Leapman et al. 1982) The observed differences in this ratio between the PEY and FY spectra can point to real differences in the chemical state of the transition metal ion in the surface as compared to the bulk. 
The partial electron yield Co L edge spectrum matches the total electron yield results of Yoon et al. (2002), but the fluorescence yield data show peak area differences. This may indicate an increased occupancy of Co $3 \mathrm{~d}$ states in the bulk as compared to the surface. Fluorescent self absorption may also play a role in reducing peak heights.

As in ex situ soft X-ray spectroscopy at the oxygen K edge, the present samples are vulnerable to oxygen vacancies near the surface of each cathode particle. However, the vacancy concentration at room temperature will be too low to affect the spectrum.

Solid state pellet batteries described in the present work are especially useful for in situ surface sensitive techniques. While this paper demonstrates the viability of direct soft X-ray spectroscopy on $\mathrm{LiCoO}_{2}$, the novel cell design allows any powdered electrode material to be studied under in situ conditions. Also, in situ XRD is feasible with some modifications. Thin film solid state batteries also have potential for in situ measurements. However, thin film batteries are limited to cathode compositions which can be deposited in thin film form and heat treated while attached to a substrate, and the anode layer may be concealed. Pellet batteries can incorporate any powdered cathode, including sol-gel-derived ceramics, and the cell can be reversed for direct spectroscopic measurements on the anode.

\section{Conclusions}

An all-solid-state, packaging-free spectroelectrochemical cell has been designed to enable simultaneous, in situ surfacesensitive and bulk-sensitive soft X-ray absorption measurements together with mass spectroscopic measurements. The soft X-ray design allows studies complementary to those carried out at hard X-ray energies.

Fluorescence yield data, for the first time, shows that in $\mathrm{LiCoO}_{2}, \mathrm{O}$ and $\mathrm{Co}$ experience different electronic environments in the bulk as compared to the surface. This difference is a key to understanding electrochemical reactions in $\mathrm{LiCoO}_{2}$ particles. Additionally, PEY spectra show that the flow of current has a transient effect on the electronic structure of oxygen. These changes may affect the evolution of oxygen gas.

The present design allows both O K edge and transition metal L edge NEXAFS spectra to be obtained with a single sample at a soft X-ray synchrotron beamline. While previous in situ methods only measured the energy of the transition metal $4 \mathrm{p}$ orbitals, the current method measures the transition metal $3 \mathrm{~d}$ orbitals and the $\mathrm{O} 2 \mathrm{p}$ orbitals, which form molecular orbitals in Li battery cathodes. In situ soft X-ray NEXAFS using this battery design can, therefore, detect the occupancies of all the relevant molecular orbitals in transition metal oxide intercalation cathodes.

Figure 1 a) The sample holder b) an enlarged cutaway view of the solid state battery

Figure 2 Normalized Fluorescence Yield and Partial Electron Yield NEXAFS spectra of $\mathrm{LiCo}_{\mathrm{O} 2}$ at the a) $\mathrm{O} \mathrm{K}$ edge, $3.1 \mathrm{~V}$ and b) Co L edge, $2.5 \mathrm{~V}$

Figure 3 Partial Electron Yield NEXAFS at the O K edge at 1.9V, 2.3V, and 3.1V

Acknowledgements The planetary ball mill was provided by Prof. Thadhani of the Georgia Institute of Technology. Certain commercial names are mentioned in this manuscript for purposes of example and do not constitute an endorsement by the National Institute of Standards and Technology. 


\section{References}

Alamgir F.M., Strauss E., Greenbaum S., Whitacre J.F., Kao C.-C., Neih S., (2005) Journal of the Electrochemical Society 152, A 845

Ceder G., Chiang Y.-M. , Sadoway D.R., Ayindol M.K., Y.-I. Jang \& Huang B., (1998) Nature 392, 694

Dahn J.R., Fuller E.W., Obrovac M., von Sacken U., (1994) Solid State Ionics 69, 265

Deb A., Bergmann U., (2004) J.S.R. 11, 497

de Groot, F.M.F., Grioni M., Fuggle J.C., Ghijsen J., Sawatzky G.A., Petersen H., (1989) Physical Review B 40, 5715

Hayashi A., Hama S., Morimoto H., Tatsumisago M., Minami T., (2001) J. Am. Ceram. Soc. 84, 477

Koikawa M., Gotoh M., Ôkawa H., Kida S., Kohzuma T., (1989) J. CHEM. SOC. DALTON TRANS., 1613

Leapman R.D., Grunes L.A., Fejes (1982) P.L., Phys. Rev. B 26 p614

Montoro L.A., Abbate M., Rosolen J.M., (2000) Electrochemical and Solid State Letters 3, 410

Pitawala H.M.J.C., Dissenanayake M.A.K.L., Seneviratne V.A., Mellander B.-E., Albinson I. (2008) J. Solid State Electrochemistry 12 p783

Tarascon J.-M., Armand M., (2001) Nature 414, 359

Uchimoto Y., Sawada H., Yao T., (2001) J.S.R. 8, 872

Yoon W.-S., Kim K.-B., Kim M.-G., Lee M.-K., Shin H.-J., Lee J.-M., Lee J.-S., Yo C.-H., (2002) J. Phys. Chem. B, 106, 2526

Yoon W.-S., Balasubramanian M., Yang X.-Q., Fu Z., Fischer D.A., McBreen J., (2004) Journal of the Electrochemical Society 151, A246

Yoon W.-S., Chung K., McBreen J., Fischer D.A., Yang S., (2007) Journal of Power Sources 174, 1015 


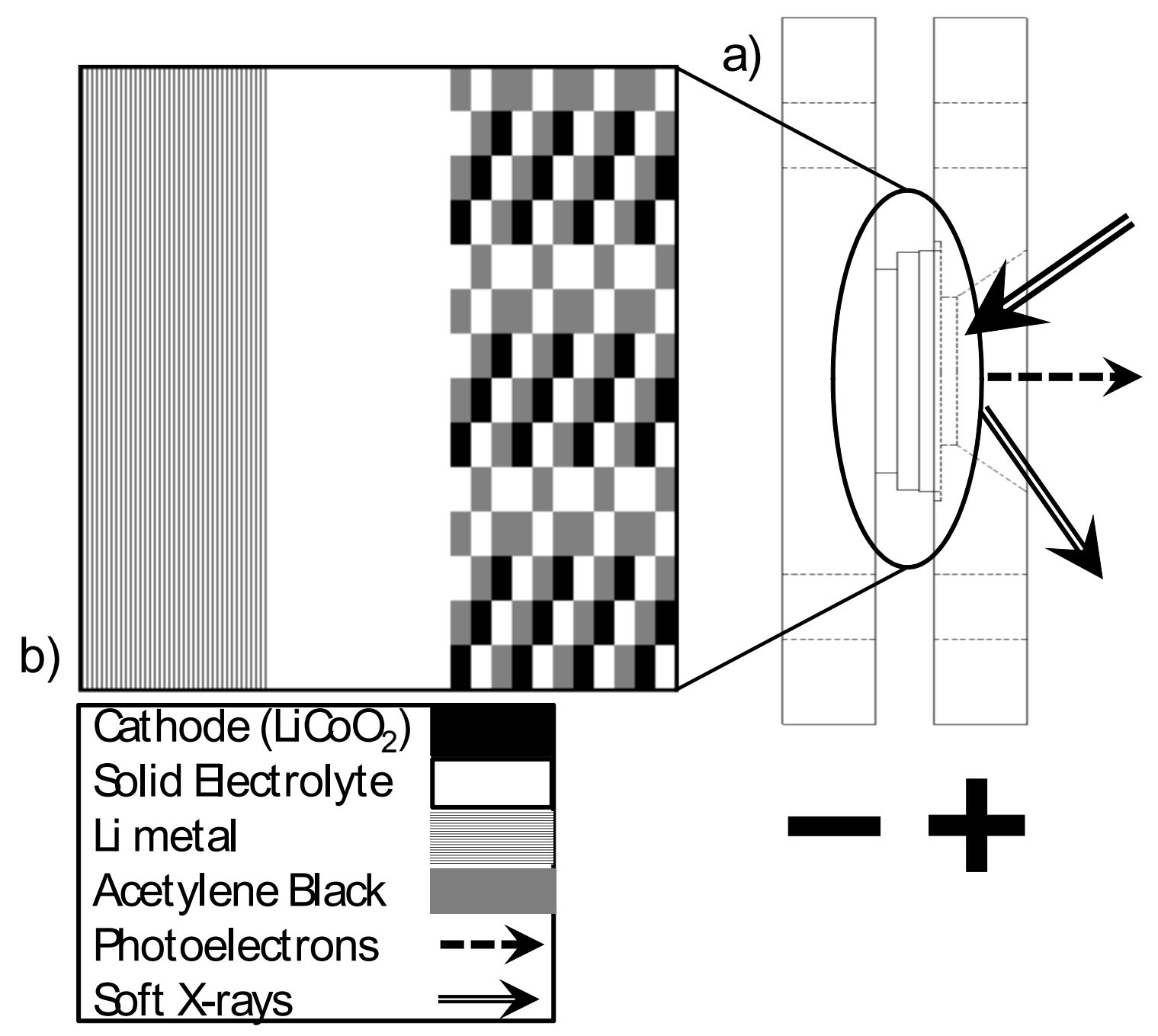

Figure 1 

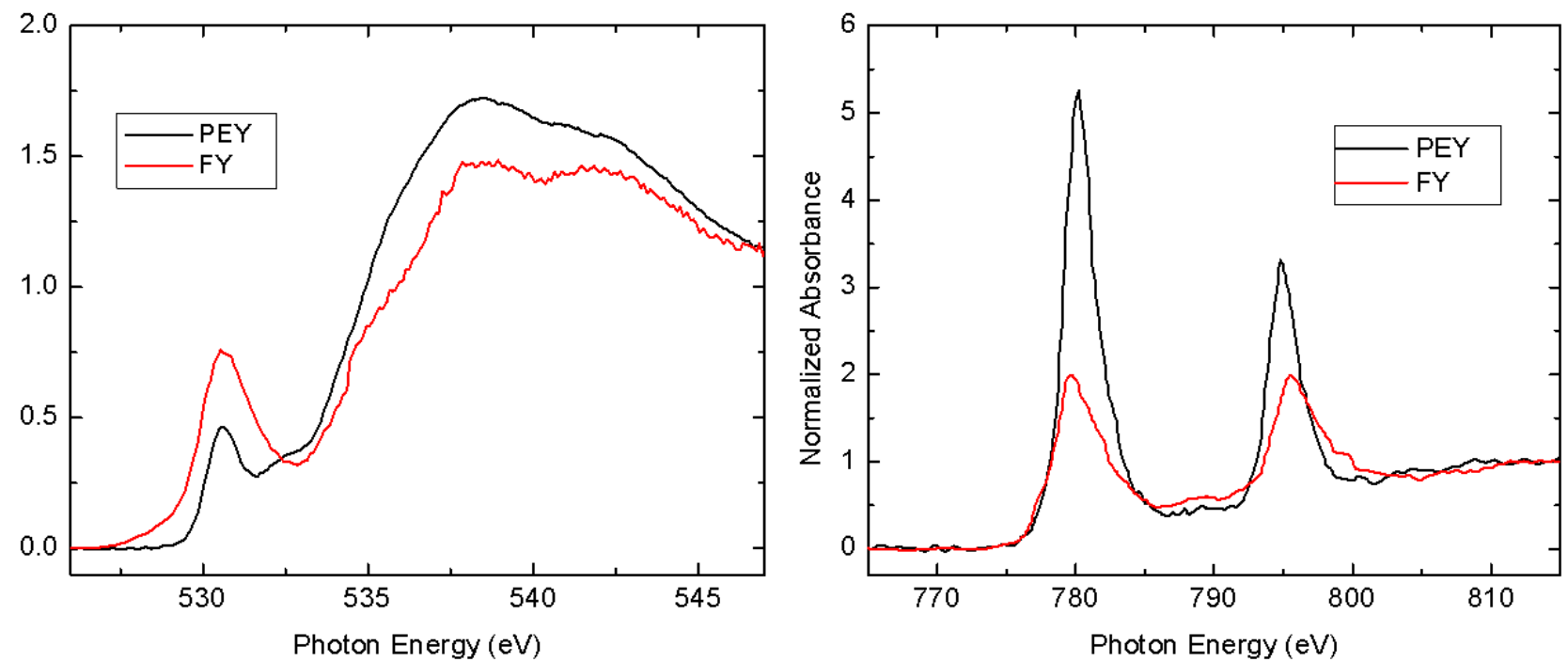

Figure 2 


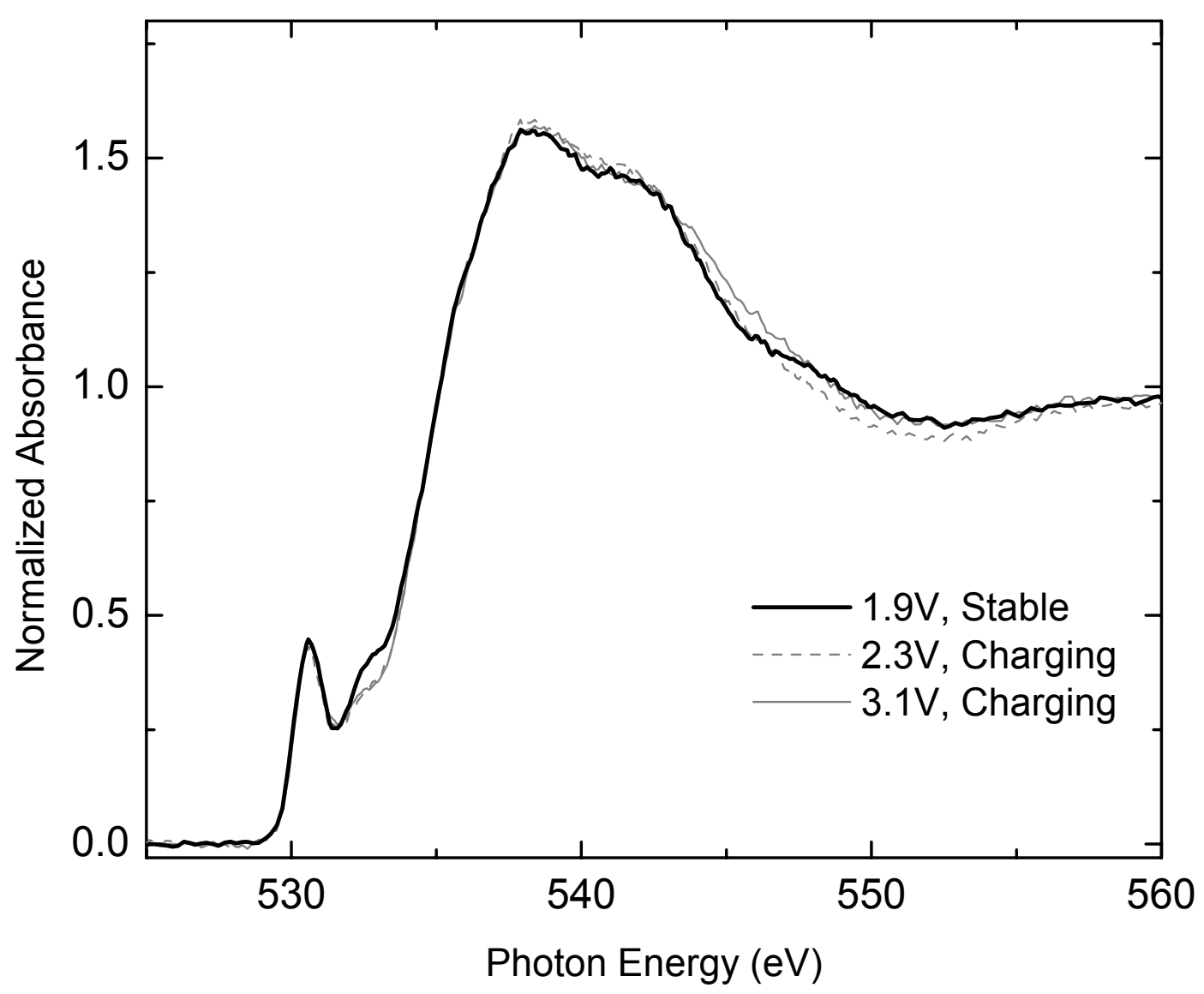

Figure 3 